\title{
Safety and Efficacy of Autologous Skeletal Myoblast Sheets (TCD-51073) for the Treatment of Severe Chronic Heart Failure Due to Ischemic Heart Disease
}

\author{
Yoshiki Sawa, MD, PhD; Yasushi Yoshikawa, MD; Koichi Toda, MD, PhD; \\ Satsuki Fukushima, MD, PhD; Kenji Yamazaki, MD, PhD; Minoru Ono, MD, PhD; \\ Yasushi Sakata, MD, PhD; Nobuhisa Hagiwara, $\mathrm{MD}, \mathrm{PhD}$; \\ Koichiro Kinugawa, MD, PhD; Shigeru Miyagawa, MD, PhD
}

\begin{abstract}
Background: Poor survival outcomes for patients with severe heart failure (HF) and the donor shortage for heart transplantation warrant the development of myocardial regenerative therapy. We performed a multicenter, phase II study to evaluate the safety and efficacy of autologous skeletal myoblast sheets (TCD-51073).

Methods and Results: In 3 study sites, we enrolled 7 patients with severe chronic HF due to ischemic heart disease despite maximal therapy, all of whom underwent transplantation of TCD-51073. No serious arrhythmia was reported, and no changes were noted in the frequency of ventricular extrasystole frequency. The primary efficacy endpoint of the change in left ventricular ejection fraction (LVEF) on gated blood-pool scintigraphy at 26 weeks after transplantation showed that 5 subjects were responders (classified as "improved" or "unchanged"). In addition, LVEF on echocardiography improved over time, with a change in LVEF of $7.1 \pm 2.8 \%$ at 26 weeks posttransplantation. Among the 7 subjects, 6 showed improvement in New York Heart Association functional class by at least 1 class. The 6-min walk distance was $410.1 \pm 136.1 \mathrm{~m}$ before transplantation and $455.4 \pm 103.7 \mathrm{~m}$ at 26 weeks after transplantation.

Conclusions: This study demonstrated the feasibility and safety of the transplantation of TCD-51073 in the patients with severe chronic HF due to ischemic heart disease, suggesting that TCD-51073 might maintain or improve cardiac function, symptoms, and physical function. (Circ J 2015; 79: 991-999)
\end{abstract}

Key Words: Heart failure; Multicenter study; Myoblast sheets; Regenerative therapy

$\mathbf{O}$ ver the past 20 years or so, the treatment of chronic heart failure (HF) has been progressed by use of drug therapies such as $\beta$-blockers, angiotensin-converting enzyme inhibitors (ACEIs), angiotensin II receptor blockers (ARBs), and aldosterone antagonists, or device therapy such as cardiac resynchronization therapy. Hence, clinical outcomes of patients with chronic HF are now significantly better, but patients with severe chronic HF who do not respond well to standard drug therapies still have poor outcomes. ${ }^{1}$ In addition, although patients with end-stage severe HF benefit from heart transplantation or left ventricular assist system (LVAS) implantation, ${ }^{2}$ these procedures are indicated for only a limited number of such patients worldwide where the number of heart transplant donors is limited. Thus, the therapeutic strategies available for patients with severe HF are still limited and new treatments need to be developed.

Since 15 years ago, treatment of heart disease with the use of patients' own somatic cells has been reported. ${ }^{3}$ Among these treatments, using autologous skeletal myoblasts has been investigated in clinical trials, mainly in Western countries, in which cell transplantation was performed using myocardial injection during a surgical procedure through a thoracotomy, such as coronary artery bypass grafting (CABG) or LVAS implantation, ${ }^{4}$ or using myocardial injection of cells through a cardiac catheter. ${ }^{5}$ However, results from a European phase II clinical study (MAGIC trial) demonstrated that transplantation

Received March 1, 2015; revised manuscript received March 26, 2015; accepted April 1, 2015; released online April 24, 2015 Time for primary review: 15 days

Department of Cardiovascular Surgery (Y. Sawa, Y.Y., K.T., S.F., S.M.), Department of Cardiovascular Medicine (Y. Sakata), Osaka University Graduate School of Medicine, Suita; Department of Cardiovascular Surgery (K.Y.), Department of Cardiology (N.H.), Tokyo Women's Medical University, Tokyo; and Department of Thoracic Surgery (M.O.), Department of Therapeutic Strategy for Heart Failure (K.K.), Graduate School of Medicine, University of Tokyo, Tokyo, Japan

This paper was presented at the $79^{\text {th }}$ Annual Scientific Meeting of the Japanese Circulation Society, Late Breaking Clinical Trials 4-6 (April 26, 2015, Osaka, Japan).

Mailing address: Yoshiki Sawa, Professor, MD, Department of Cardiovascular Surgery, Osaka University Graduate School of Medicine, 2-2 Yamadaoka, Suita 565-0871, Japan. E-mail: sawa@surg1.med.osaka-u.ac.jp

ISSN-1346-9843 doi:10.1253/circj.CJ-15-0243

All rights are reserved to the Japanese Circulation Society. For permissions, please e-mail: cj@j-circ.or.jp 
of autologous skeletal myoblasts was less effective than $\mathrm{CABG}$ as a control procedure. ${ }^{6}$

We developed an autologous skeletal myoblast sheet by using the cell sheet engineering approach developed by Okano et al of Tokyo Women's Medical University, Japan. ${ }^{10}$ Following a number of nonclinical studies, ${ }^{7-11}$ we conducted the firstin-human study at Osaka University in subjects with dilated cardiomyopathy who had received an LVAS, and we confirmed the feasibility and safety of the cell sheet, with successful weaning from the LVAS in 2 subjects. ${ }^{12} \mathrm{We}$ also conducted a phase I study in subjects with severe ischemic cardiomyopathy and dilated cardiomyopathy (UMIN ID; 000003273), and confirmed the safety and feasibility of the sheet in these cohorts. The results supported investigation of the practical use of autologous skeletal myoblast sheets on a commercial basis, leading to the development of TCD-51073 (Terumo Corporation, Tokyo, Japan).

We, therefore, conducted an exploratory, prospective, multicenter, uncontrolled, open-label phase II study of TCD51073 in subjects with severe chronic HF due to ischemic heart disease, which we designed (1) to validate the method of efficacy evaluation, (2) to collect safety information, and (3) to confirm that transplantation or other procedures at multiple medical institutions was successfully conducted.

\section{Methods}

This study was conducted in accordance with the ethical principles of the Declaration of Helsinki, Japanese Pharmaceutical Affairs Law, and Good Clinical Practice. The study was approved by the institutional review board at each study site, and all of the subjects provided prior informed consent to participate in the study.

\section{Patients and Procedures}

Patients This study included patients with ischemic heart disease who had impaired left ventricular systolic function and remained in HF status despite maximal oral therapy, including digitalis, diuretics, ACEIs, ARBs, $\beta$-blockers, aldosterone antagonists, and oral inotropic agents, and thus were at risk of worsening HF despite standard-of-care therapy. The inclusion criteria were: (1) patients who had chronic ischemic heart disease; (2) patients in New York Heart Association (NYHA) class III or IV HF; (3) patients who remained in HF status despite maximal oral therapy, including digitalis, diuretics, ACEIs, ARBs, $\beta$-blockers, aldosterone antagonists, and oral inotropic agents; (4) patients aged 20 years or older at the time of consent; (5) patients who were at risk of worsening $\mathrm{HF}$ despite standard-of-care therapy (eg, CABG, mitral valvuloplasty, LV restoration, cardiac resynchronization therapy, and percutaneous coronary intervention) conducted at least 3 months earlier; and (6) patients who had a LV ejection fraction (LVEF) $\leq 35 \%$ on resting echocardiography. The exclusion criteria were: (1) patients with evidence of skeletal muscle disease; (2) patients undergoing thyroid hormone treatment; (3) patients with infectious diseases (eg, infections caused by the human immunodeficiency virus, hepatitis B virus, hepatitis $\mathrm{C}$ virus, and human T cell leukemia virus type 1); (4) patients who remained in shock because of worsening HF; (5) patients with irreversible non-cardiac organ failure; (6) patients with any malignancy; (7) patients who were pregnant or possibly pregnant; (8) patients with a history of alcoholism or drug addiction within 6 months before the day of consent; and (9) patients with severe pulmonary hypertension.

Procedure At each study site, skeletal muscle (required amount, 2-5g) was harvested from the vastus medialis muscle of the subject by aseptic technique, under general anesthesia and with endotracheal intubation. In the Cell Processing Center of Terumo Corporation, skeletal myoblasts were isolated from the harvested skeletal muscle by enzymatic digestion in TrypLE Select ${ }^{\mathrm{TM}}$ (Thermo Fisher Scientific Inc, MA, USA) containing collagenase, expanded in culture in MCDB103 medium with fetal bovine serum for approximately 4 weeks, and cryopreserved. At least 7 weeks after the harvest of the skeletal muscle, the skeletal myoblasts were seeded at $6.0 \times 10^{7}$ cells per dish and incubated on temperature-responsive culture dishes $(10 \mathrm{~cm}$ in diameter) to form a skeletal myoblast sheet (TCD-51073), as described previously. ${ }^{7}$ Transplantation of TCD-51073 was performed through a left thoracotomy under general anesthesia, and 5 sheets of TCD-51073 (representing $3 \times 10^{8}$ cells) were transplanted onto a large area extending from the anterior wall to the lateral wall of the left ventricle. No other concomitant cardiac surgery, such as CABG or mitral valve repair, were performed.

\section{Evaluation Methods}

Study Protocol Evaluation for the study was performed up to 26 weeks after transplantation. A 2-year follow-up study is ongoing, which was begun after the evaluation period. Observations included physical examination (subjective and objective), NYHA classification, specific activity scale (SAS) score, and concomitant medications. Examinations included vital signs, body weight, laboratory tests, chest radiography, cardiac computed tomography (CT), echocardiography, resting standard 12-lead ECG, 24-h Holter ECG monitoring, 6-min walk test, cardiopulmonary exercise test (CPX), and gated equilibrium blood-pool scintigraphy. Myocardial perfusion single-photon emission CT and coronary angiography were performed for eligibility screening.

Primary Endpoint The primary endpoint was the change in LVEF on gated equilibrium blood-pool scintigraphy from pretransplantation to 26 weeks posttransplantation. This endpoint was chosen based on the advice provided by the Pharmaceuticals and Medical Devices Agency in Japan.

Secondary Endpoints The prespecified secondary endpoints included success or failure of the transplantation, LVEF, LV end-diastolic volume index, LV end-systolic volume index, NYHA classification, SAS, 6-min-walk distance (6MWD), peak oxygen uptake (peak $\dot{\mathrm{VO}}_{2}$ ), anaerobic threshold (AT), B-type natriuretic peptide (BNP), and volumetric analysis of echocardiography performed on the long axes of apical 2- and 4-chamber views using the modified Simpson method.

Safety Endpoints Safety endpoints included arrhythmia, adverse events (AEs) and adverse drug reactions (ADRs) other than arrhythmia, serious arrhythmia (requiring inpatient hospitalization or prolonging the existing hospitalization for treatment or monitoring) and serious AEs (SAEs), AEs associated with harvest of skeletal muscle tissues, changes in vital signs over time, and changes in routine hematology and clinical chemistry parameters over time.

Study Administrative Structure The Case Adjudication Committee, which consisted of 2 independent cardiologists and 1 independent cardiac surgeon, was established to provide advice on the determination of patient eligibility and whether the study should be continued, as well as to evaluate efficacy. In addition, the Data and Safety Monitoring Committee, which consisted of 1 cardiac surgeon and 1 medical statistician, was established to review safety data from this study at appropriate intervals or at necessary times to provide recommendations 
for continuation, modification, or termination of the study to the sponsor, as necessary. The patients were registered at the patient registration center (EPS Corporation, Tokyo, Japan), and cardiac CT scans were analyzed at the core laboratory (TITAN Inc, Tokyo, Japan).

\section{Statistical Analysis}

Primary Endpoint The patients were classified according to the change in LVEF from pretransplantation to 26 weeks posttransplantation as "improved" $(\triangle \mathrm{LVEF} \geq 5 \%)$, "unchanged" $(5 \%>\Delta \mathrm{LVEF}>-3 \%)$, or "worsened" $(-3 \% \geq \Delta \mathrm{LVEF})$. A responder was defined as a subject whose LVEF was classified as improved or unchanged, and the number of responders was determined.

Secondary Endpoints For continuous variables, summary statistical indexes (mean, standard deviation [SD and 2-sided $95 \%$ confidence interval $[\mathrm{CI}]$ for the mean, maximum, median, minimum, and number of subjects) were calculated. For discrete variables, frequency distribution tables were constructed.

Safety Endpoints For arrhythmia, and AEs and ADRs other than arrhythmia, the numbers of events and the subjects with events were determined. If an event was assessed as an ADR, the numbers of such events and the subjects with such events were determined. For significant AEs and SAEs, as well as AEs associated with harvest of skeletal muscle tissues, the numbers of events and the subjects with events were determined. For vital signs, routine hematology, and clinical chemistry parameters, summary statistical indexes (mean, SD, and 2-sided $95 \%$ CI for the mean, maximum, median, minimum, and number of subjects) were calculated and the time course of the changes was determined.

\section{Results}

\section{Classification and Disposition of Subjects}

From 3 Japanese study sites, we enrolled 7 patients with severe chronic HF due to ischemic heart disease who continued to be in NYHA class III or higher and have an LVEF $\leq 35 \%$ despite maximal therapy between May 2012 and October 2013 (Osaka University Hospital, 4 patients; Tokyo Women's Medical University Hospital, 2 patients; University of Tokyo Hospital, 1 patient). Of the 8 initial subjects who provided informed consent and reviewed by the Case Adjudication Committee, 1 was considered ineligible. All 7 eligible subjects received transplantation of TCD-51073 and completed the study without discontinuations.

\section{Baseline Characteristics of the Patients}

The baseline characteristics of the enrolled subjects are shown in Table 1 . The mean age at eligibility screening was 56.3 \pm 13.2 years (range, 35-71 years), and all of them were male. All the subjects had undergone percutaneous coronary intervention or CABG for ischemic heart disease but presented with symptoms of severe HF, were in NYHA class III, and had a LVEF of $29 \pm 3.7 \%$ ( $<30 \%$ in 3 subjects) on echocardiography. All the subjects were identified as having no indication for further revascularization. In addition, 2 subjects had an implantable cardioverter defibrillator or cardiac resynchronization therapy defibrillator, and 1 subject had undergone mitral valvuloplasty. Mitral regurgitation was reported in 6 subjects, as assessed pretransplantation by echocardiography. One subject who had a history of NYHA class IV symptoms was listed as a status 2 cardiac transplantation candidate by the Japan Organ Transplant Network.

\begin{tabular}{|c|c|}
\hline \multicolumn{2}{|l|}{ Demographics } \\
\hline No. of patients & 7 \\
\hline Age, years (mean $\pm S D)$ & $56.3 \pm 13.2$ \\
\hline$>65$ years & $2(28.6 \%)$ \\
\hline Male sex & $7(100 \%)$ \\
\hline Body weight $(\mathrm{kg})[$ mean $\pm S D]$ & $70.3 \pm 9.5$ \\
\hline Height $(\mathrm{cm})[$ mean \pm SD] & $168.9 \pm 6.0$ \\
\hline Body surface area $\left(\mathrm{m}^{2}\right)[$ mean $\pm S D]$ & $1.8 \pm 0.1$ \\
\hline \multicolumn{2}{|l|}{ Cardiac function } \\
\hline NYHA functional class III & $7(100 \%)$ \\
\hline LVEF (echocardiography) [mean \pm SD] & $29.3 \pm 3.7$ \\
\hline \multicolumn{2}{|l|}{ Risk factor } \\
\hline Hypertension & $3(42.9 \%)$ \\
\hline Hyperlipidemia & $6(85.7 \%)$ \\
\hline Diabetes mellitus & $3(42.9 \%)$ \\
\hline Oral medication & $3(42.9 \%)$ \\
\hline Insulin & $1(14.3 \%)$ \\
\hline Smoking & $7(100 \%)$ \\
\hline Current or previous (within the past year) & $0(0.0 \%)$ \\
\hline \multicolumn{2}{|l|}{ Cardiac history } \\
\hline $\mathrm{PCl}$ or $\mathrm{CABG}$ & $7(100 \%)$ \\
\hline $\mathrm{PCl}$ & $6(85.7 \%)$ \\
\hline CABG & $6(85.7 \%)$ \\
\hline Pacemaker implant & $0(0.0 \%)$ \\
\hline ICD implant & $1(14.3 \%)$ \\
\hline CRT implant & $0(0.0 \%)$ \\
\hline CRT-D implant & $1(14.3 \%)$ \\
\hline Valve surgery & $1(14.3 \%)$ \\
\hline Left ventricular reconstruction & $0(0.0 \%)$ \\
\hline IABP & $1(14.3 \%)$ \\
\hline LVAD & $0(0.0 \%)$ \\
\hline Myocardial infarction & $6(85.7 \%)$ \\
\hline Nonsustained VT & $2(28.6 \%)$ \\
\hline Atrial fibrillation or flutter & $3(42.9 \%)$ \\
\hline Mitral regurgitation & $6(85.7 \%)$ \\
\hline \multicolumn{2}{|l|}{ Medication } \\
\hline ACEI or ARB & $6(85.7 \%)$ \\
\hline ACEI & $5(71.4 \%)$ \\
\hline ARB & $1(14.3 \%)$ \\
\hline$\beta$-blocker & $7(100 \%)$ \\
\hline Aldosterone receptor antagonist & $5(71.4 \%)$ \\
\hline Diuretics & $6(85.7 \%)$ \\
\hline Inotropic & $2(28.6 \%)$ \\
\hline Antiplatelet & $6(85.7 \%)$ \\
\hline Warfarin & $5(71.4 \%)$ \\
\hline Amiodarone & $4(57.1 \%)$ \\
\hline Hypoglycemic & $3(42.9 \%)$ \\
\hline Statins & $6(85.7 \%)$ \\
\hline
\end{tabular}

ACEl, angiotensin-converting enzyme inhibitor; ARB, angiotensin II receptor blocker; CABG, coronary artery bypass grafting; CRT, cardiac resynchronization therapy; CRT-D, cardiac resynchronization-defibrillator therapy; IABP, intra-aortic balloon pump; ICD, implantable cardioverter defibrillator; LVAD, left ventricular assist device; LVEF, left ventricular ejection fraction; NYHA, New York Heart Association; $\mathrm{PCl}$, percutaneous coronary intervention; SD, standard deviation; VF, ventricular fibrillation; VT, ventricular tachycardia. 
Table 2. Summary of Adverse Events Among Patients Undergoing Cell Sheet Transplantation

\begin{tabular}{|c|c|c|c|c|c|c|c|c|}
\hline \multirow[b]{2}{*}{ Adverse event (MedDRA SOC) } & \multicolumn{6}{|c|}{ Not related* } & \multirow{2}{*}{$\begin{array}{l}\text { Cannot be } \\
\text { ruled out }\end{array}$} & \multirow[b]{2}{*}{ Total } \\
\hline & $\begin{array}{l}\text { Underlying } \\
\text { disease }\end{array}$ & Surgery & $\begin{array}{l}\text { Concomitant } \\
\text { medication }\end{array}$ & $\begin{array}{l}\text { Patient's } \\
\text { condition }\end{array}$ & $\begin{array}{l}\text { Concurrent } \\
\text { conditions }\end{array}$ & Other & & \\
\hline $\begin{array}{l}\text { Congenital blood and lymphatic system } \\
\text { disorders }\end{array}$ & & & & 1 & 1 & & & 2 \\
\hline Cardiac disorders & 4 & 3 & & & & & 1 & 8 \\
\hline Gastrointestinal disorders & & 1 & 1 & & & 2 & & 3 \\
\hline $\begin{array}{l}\text { General disorders and administration } \\
\text { site conditions }\end{array}$ & 1 & 1 & & 1 & & & & 3 \\
\hline Hepatobiliary disorders & & 1 & & 1 & & 1 & & 3 \\
\hline Infections and infestations & & 5 & & 1 & & & & 6 \\
\hline $\begin{array}{l}\text { Injury, poisoning, and procedural compli- } \\
\text { cations }\end{array}$ & & 9 & & & & 1 & & 10 \\
\hline Investigations & 1 & 3 & 3 & & & 4 & & 11 \\
\hline Metabolism and nutrition disorders & & 2 & 4 & & & & & 6 \\
\hline $\begin{array}{l}\text { Musculoskeletal and connective tissue } \\
\text { disorders }\end{array}$ & & & 1 & & & 2 & & 3 \\
\hline $\begin{array}{l}\text { Neoplasms benign, malignant, and } \\
\text { unspecified (including cysts and polyps) }\end{array}$ & & & & & 1 & & & 1 \\
\hline Nervous system disorders & & & 1 & & & & & 1 \\
\hline Psychiatric disorders & & 1 & & & & & & 1 \\
\hline Renal and urinary disorders & & 4 & & & & 1 & & 5 \\
\hline $\begin{array}{l}\text { Reproductive system and breast disor- } \\
\text { ders }\end{array}$ & & & 1 & & & & & 1 \\
\hline $\begin{array}{l}\text { Respiratory, thoracic, and mediastinal } \\
\text { disorders }\end{array}$ & & 4 & & & & & & 4 \\
\hline Skin and subcutaneous tissue disorders & & 4 & & & & & & 4 \\
\hline Vascular disorders & & 2 & & & & & & 2 \\
\hline
\end{tabular}

${ }^{*}$ If there are 2 or more reasons, each is counted.

\begin{tabular}{|ccccc|}
\hline \multicolumn{4}{|c|}{ Table 3. Summary of Arrhythmia Among Patients Undergoing Cell Sheet Transplantation } \\
Patient ID & $\begin{array}{c}\text { Adverse event } \\
\text { (MedDRA LLT) }\end{array}$ & $\begin{array}{c}\text { Post Tx } \\
\text { (days) }\end{array}$ & $\begin{array}{c}\text { Relationship to } \\
\text { study drug }\end{array}$ & Reason for "not related" \\
T01-01 & Nonsustained VT & 6 & Not related & Underlying disease \\
T03-01 & Atrial fibrillation & 2 & Not related & Underlying disease \\
T01-04 & Nonsustained VT & 4 & Not related & $\begin{array}{c}\text { Underlying disease, Possibility of } \\
\text { concomitant medications }\end{array}$ \\
T02-02 & Atrial flutter & 4 & Not related & Surgery \\
& Atrial fibrillation & 5 & Not related & Surgery \\
T01-05 & Ventricular extrasystoles & 1 & Not related & $\begin{array}{c}\text { Underlying disease, Possibility of } \\
\text { concomitant medications, Surgery }\end{array}$ \\
\hline
\end{tabular}

Abbreviation as in Table 1.

\section{Surgical Procedure}

Harvest of the skeletal muscle and cell culture were successfully performed in all the enrolled subjects, who were transplanted with 5 sheets of TCD-51073. The proportion of skeletal myoblasts (CD56-positive cells) in the transplanted TCD-51073 was $\geq 60 \%$, with a cell viability $\geq 75 \%$, indicating evidence of fusion ability in all subjects. This product also complied with sterility, bacterial endotoxins, and mycoplasma tests.

\section{Safety Evaluation}

The AEs reported in this study are listed in Table 2. AEs associated with the harvest of the skeletal muscle were observed in 2 of the 7 subjects and included 2 events of wound complication in 2 subjects and 1 event of postprocedural swelling in
1 subject. These AEs all resolved without sequelae within 1-14 days. After TCD-51073 transplantation, 74 AEs were observed in the total group. Among these AEs, the most commonly observed were wound complications in 4 subjects, hypokalemia in 3 , and postoperative fever in 3 . No subject except those with SAEs, as described next, required specific treatment.

Serious arrhythmia, which required inpatient hospitalization or prolonged existing hospitalization for treatment or monitoring, was defined as a significant AE in this study, but no cases occurred. In contrast, 6 arrhythmia events occurred in 5 subjects, including 2 events of ventricular arrhythmia in 2 subjects, 1 event of ventricular extrasystole in 1 subject, 2 events of atrial fibrillation in 2 subjects, and 1 event of atrial flutter in 1 subject (Table 3). Among the 2 subjects with 


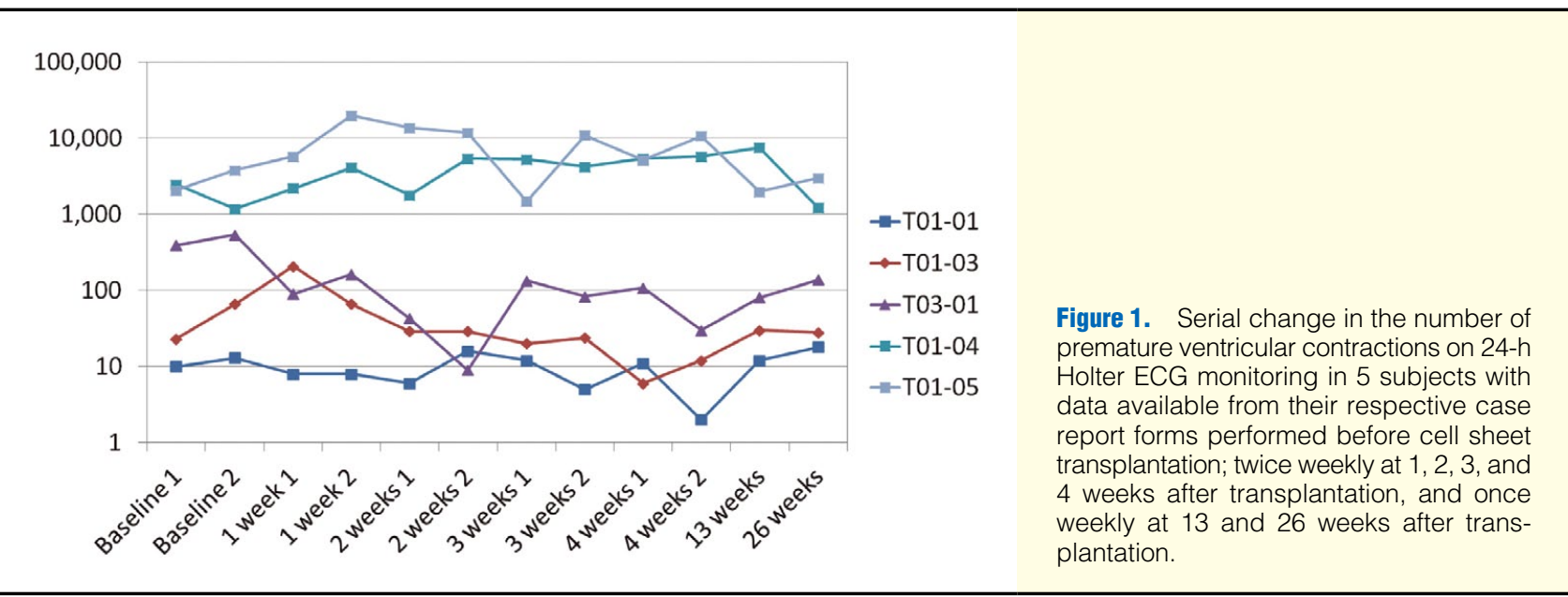

nonsustained ventricular arrhythmia, in subject T01-01 with existing nonsustained ventricular tachycardia, the nonsustained ventricular arrhythmia resolved with drug therapy on the day of onset. In subject T01-04, with existing multiple nonsustained ventricular tachycardias, the nonsustained ventricular arrhythmia did not resolve, but was mild and required no additional drug therapy or treatment. These arrhythmic events were all reported within 1 week after transplantation and were considered attributable to the transplantation procedure or underlying disease.

In addition, the subjects were hospitalized for 4 weeks after transplantation to further investigate whether arrhythmogenic effects were present and 24-h Holter ECG monitoring was performed before transplantation; twice weekly at 1,2, 3, and 4 weeks after transplantation; and once weekly at 13 and 26 weeks after transplantation. The ECG changes over time in each subject who received TCD-51073 transplantation showed no specific trend, with no significant change in the frequency of ventricular extrasystole (Figure 1).

In this study, 3 SAEs occurred in 3 subjects, comprising colon cancer (subject T02-01, 182 days posttransplantation), prolonged HF (subject T01-04, 31 days posttransplantation), and aggravated HF (subject T03-01, 49 days posttransplantation). The colon cancer was diagnosed as a primary cancer based on pathological findings and was considered unrelated to TCD-51073. This subject underwent right hemicolectomy at 273 days posttransplantation and subsequently recovered. The prolonged HF was attributed to surgery, inappropriate drug administration, and the subject's lifestyle, and was considered unrelated to the transplanted TCD-51073. This subject was discharged from hospital at 141 days posttransplantation, but was re-hospitalized at 159 days posttransplantation. The patient was subsequently discharged from hospital at 172 days posttransplantation, and recovered. The relationship between the event of aggravated HF and the transplanted TCD-51073 was considered unknown. This subject was a Status 2 patient registered for heart transplantation and repeatedly hospitalized and discharged 4 times until 26 weeks posttransplantation, with drugs added and/or modified because of the aggravated HF during 3 of the hospitalizations. For all episodes of hospitalization, the subject recovered with intravenous infusion of inotropic agents and diuretics or modification of diuretics for fluid control and was discharged from hospital.

In the vital signs, physical examination results, and clinical laboratory test results, no serious abnormal changes occurred

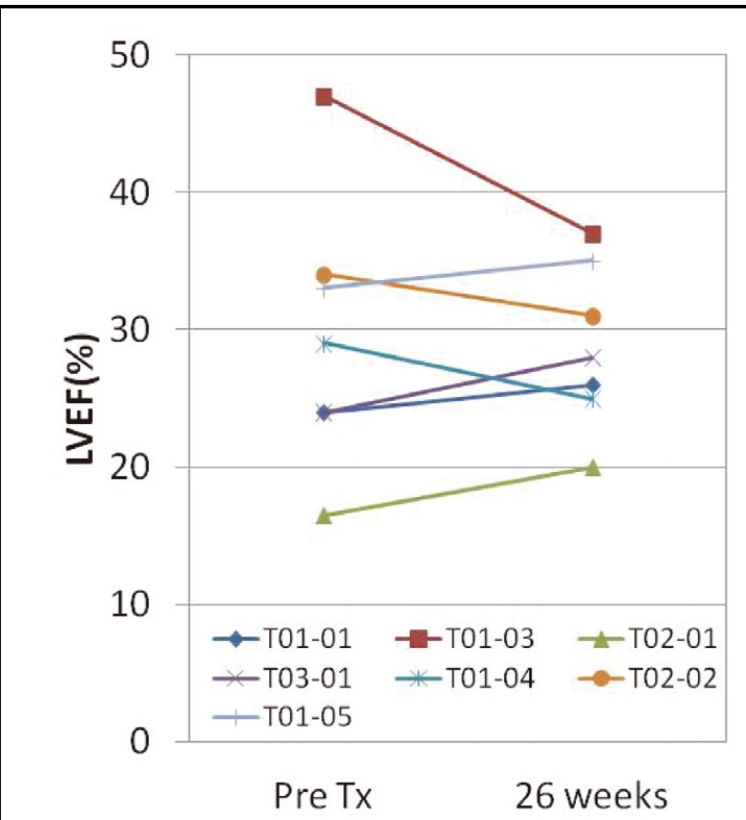

Figure 2. Change in left ventricular ejection fraction (LVEF) from pretransplantation to 26 weeks post cell sheet transplantation using gated equilibrium blood-pool scintigraphy.

in any of the subjects, although effects of the thoracotomy, and concomitant medications or changes associated with observed AEs were noted. For body weight, no serious abnormal changes occurred. No clinically significant abnormal changes or abnormal changes qualifying as SAEs were noted.

Primary Efficacy Endpoint Figure 2 shows the change in LVEF over time according to gated equilibrium blood-pool scintigraphy. The LVEF was found to be "unchanged" in 5 of the 7 subjects. A responder was defined as a subject in whom the LVEF was classified as "improved" or "unchanged" in the present study. Accordingly, 5 subjects were considered to be responders. Among the 2 subject with worsened LVEF, 1 subject (subject T01-04) received continuous administration of drugs that affected LVEF values, including intravenous inotropic agents (eg, dobutamine) and diuretics, for the management of AEs that occurred after transplantation (prolonged 
A

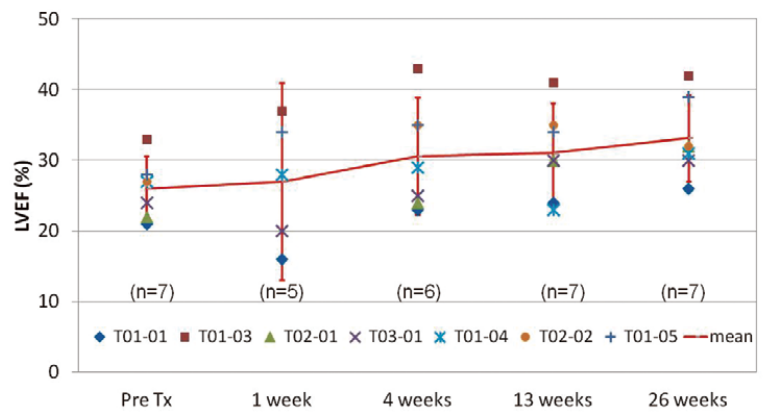

B

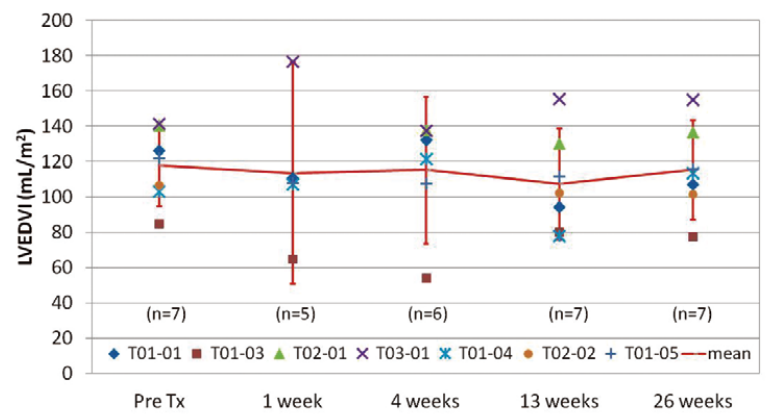

\section{C}

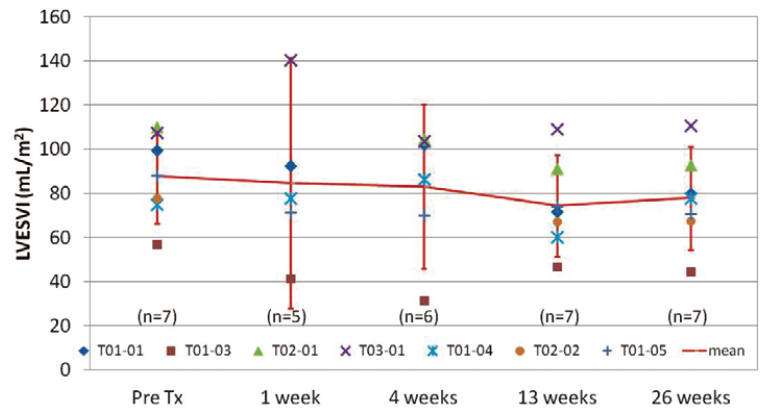

D

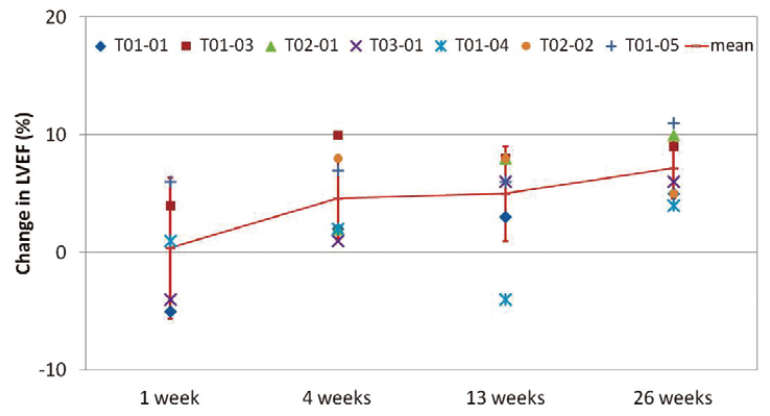

E

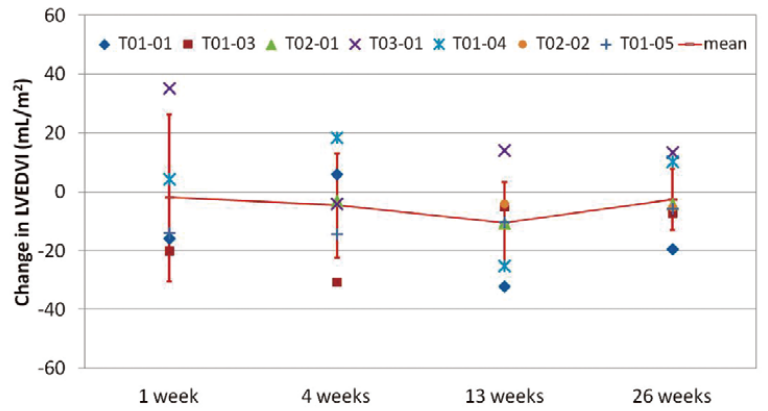

$\mathbf{F}$

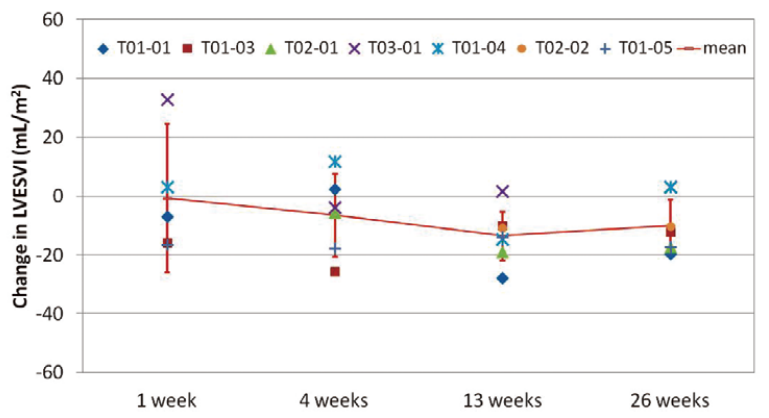

Figure 3. Serial change in echocardiographic parameters from baseline to 26 weeks after cell sheet transplantation: (A) left ventricular ejection fraction (LVEF), (B) left ventricular end-diastolic volume index (LVEDVI) and (C) left ventricular end-systolic volume index (LVESVI). Change from pretransplantation to 26 weeks posttransplantation in (D) LVEF, (E) LVEDVI and (F) LVESVI. Error bar are expressed as mean $\pm 95 \%$ confidence interval.

$\mathrm{HF}$ and renal impairment). The other subject (subject T01-03) became unwell at the time of LVEF measurement before transplantation, and decreased blood pressure associated with administration of a tracer was likely to have affected the LVEF values. These 2 patients showed an increase in LVEF on echocardiography.

Secondary Efficacy Endpoints Results of the echocardiography performed before and at $1,4,13$, and 26 weeks after transplantation showed LVEF values of $26.0 \pm 4.1 \%, 27.0 \pm 8.9 \%$, $30.6 \pm 7.4 \%, 31.0 \pm 6.3 \%$, and $33.1 \pm 5.5 \%$, respectively (Figure 3A). This is consistent with a trend toward improvement over time, with the changes in LVEF values from pretransplantation to 1, 4,13 , and 26 weeks posttransplantation being $0.4 \pm 4.8 \%$, $4.6 \pm 3.6 \%, 5.0 \pm 4.4 \%$, and $7.1 \pm 2.8 \%$, respectively (Figure 3D). In addition, an increase in LVEF values from pretransplanta- tion was observed in all subjects, with an increase $>5 \%$ from pretransplantation values in 4 subjects. The change in LVEF from pretransplantation to 26 weeks posttransplantation was $2.0 \pm 2.6 \%$ in 6 subjects in whom cardiac CT was feasible. No LVEF values could be obtained in 1 subject (subject T01-04) with worsened LVEF by gated equilibrium blood-pool scintigraphy at 26 weeks posttransplantation. In the other subject (subject T01-03), LVEF was unchanged.

Changes in NYHA class over time are shown in Figure 4A. Before transplantation, all subjects were in NYHA class III, but 2, 4, and 1 of the subjects were in NYHA class I, II, and III, respectively, at 26 weeks posttransplantation, indicating improvement in NYHA class by at least 1 class in 6 of the 7 subjects who received a transplant; 2 of the 6 subjects experienced a substantial improvement from class III to class I. 
SAS results showed an improvement of at least 1 metabolic equivalent (MET) in 3 subjects and no change in 4 subjects, from pretransplantation (Figure 4B). Subject T01-01 had an increase of at least 2 METs, from 3-4 METs pretransplantation to 6-7 METs at 26 weeks posttransplantation. Subject T01-03 had an increase of at least 2 METs, from 4-5 METs pretransplantation to 6-7 METs at 26 weeks posttransplantation.

The results of the 6MWD and CPX are shown in Table 4. The 6MWD was $410.1 \pm 136.1 \mathrm{~m}$ pretransplantation and $455.4 \pm$ $103.7 \mathrm{~m}$ at 26 weeks posttransplantation. Subjects T01-03 and T03-01 experienced increases of 170 and $214 \mathrm{~m}$, respectively, whereas subject T02-02 had a decrease of $110 \mathrm{~m}$ from pretransplantation. Peak $\mathrm{VO}_{2}$ was $13.4 \pm 5.3 \mathrm{ml} \cdot \mathrm{kg}^{-1} \cdot \mathrm{min}^{-1}$ pretransplantation and $14.6 \pm 4.9 \mathrm{ml} \cdot \mathrm{kg}^{-1} \cdot \mathrm{min}^{-1}$ at 26 weeks posttransplantation. AT was $8.7 \pm 2.3 \mathrm{ml} \cdot \mathrm{kg}^{-1} \cdot \mathrm{min}^{-1}$ pretransplantation and $9.3 \pm 2.2 \mathrm{ml} \cdot \mathrm{kg}^{-1} \cdot \mathrm{min}^{-1}$ at 26 weeks posttransplantation. In the 5 subjects in which a comparison before and after transplantation could be made, peak $\mathrm{VO}_{2}$ and AT showed a trend toward worsening in 2 subjects and no change or a trend toward improvement in 3 subjects from pretransplantation.

Subject T02-01 was diagnosed with colon cancer at the time of examination at 26 weeks posttransplantation and had physical examination findings such as shortness of breath on exertion and staggering, which may have affected the results of NYHA classification, SAS, 6MWD, and CPX.

\section{Discussion}

\section{Overview of Study Results}

This study was an exploratory study designed to evaluate the efficacy and safety of TCD-51073 in subjects presenting with severe chronic HF due to ischemic heart disease who were at risk of worsening HF despite maximal therapy. Despite the small sample size, the results demonstrated the following: (1) harvesting of the skeletal muscle and then cell culture were successfully performed in all enrolled subjects; (2) transplantation of TCD-51073 was safe and feasible in multiple medical institutions; (3) no evidence of serious arrhythmia and no other possible SAEs were observed; (4) LVEF was maintained in 5 subjects on gated equilibrium blood-pool scintigraphy and showed improvement over time on echocardiography; (5) 6 subjects showed improvement in NYHA class and almost all subjects showed symptomatic improvement. Some subjects showed improvement in exercise tolerance.

\section{Efficacy Evaluation}

This study demonstrated that 5 of the 7 subjects were respond-

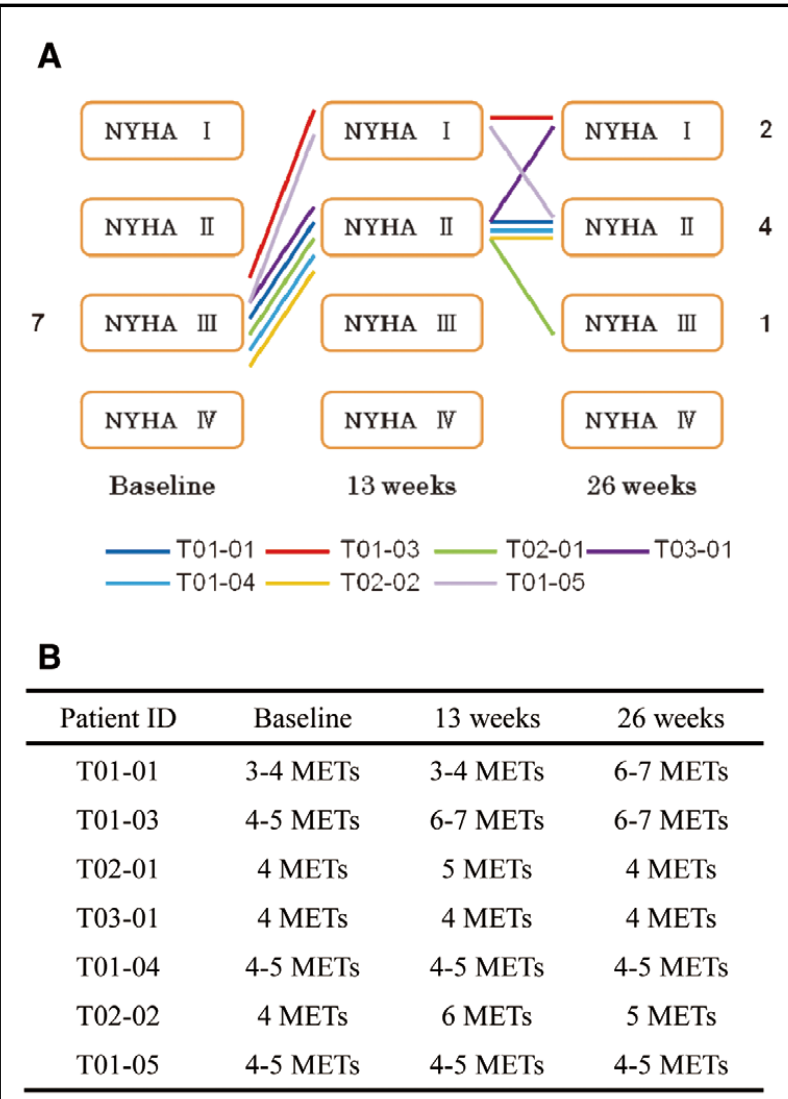

Figure 4. Serial change in New York Heart Association (NYHA) class and specific activity scale (SAS) from baseline to 26 weeks after cell sheet transplantation.

ers in the primary endpoint. Of the 2 subjects with worsened LVEF, 1 subject became unwell at the time of LVEF measurement before transplantation, and the decreased blood pressure associated with administration of a tracer was likely to have affected the LVEF values. The other subject had a variable degree of mitral regurgitation and thus was not suitable for the assessment of changes in systolic function based on LVEF. In addition, the secondary endpoint of LVEF showed improvements in cardiac function over time on echocardiography, suggesting that this product might at least be effective in maintaining cardiac function in patients with severe HF who are at risk of worsening. Overall improvement in the secondary

\begin{tabular}{|c|c|c|c|c|c|c|}
\hline \multirow{2}{*}{ Patient ID } & \multicolumn{2}{|c|}{ 6-min walk distance $(\mathrm{m})$} & \multicolumn{2}{|c|}{ Peak $\mathrm{VO}_{2}\left(\mathrm{ml} \cdot \mathrm{kg}^{-1} \cdot \mathrm{min}^{-1}\right)$} & \multicolumn{2}{|c|}{ AT $\left(\mathrm{ml} \cdot \mathrm{kg}^{-1} \cdot \mathrm{min}^{-1}\right)$} \\
\hline & Baseline & 26 weeks & Baseline & 26 weeks & Baseline & 26 weeks \\
\hline T01-01 & 485 & 520 & 19.4 & 16.5 & 10.5 & 8.6 \\
\hline T01-03 & 400 & 570 & 20.4 & 21.1 & 11.3 & 12 \\
\hline T02-01 & 486 & $462^{*}$ & 12.6 & $7.6^{*}$ & 10.1 & 7 \\
\hline T03-01 & 264 & 478 & 9.1 & 13.3 & 6.7 & 7.7 \\
\hline T01-04 & 285 & 291 & 7.8 & $-^{\star}$ & 5.4 & $-^{*}$ \\
\hline T02-02 & 640 & 530 & 11 & 14.5 & 8.3 & 11 \\
\hline T01-05 & 311 & 337 & $-^{*}$ & $-^{\star}$ & - & $-^{\star}$ \\
\hline
\end{tabular}

${ }^{*}$ No examination was performed. 
endpoint (NYHA classification) was noted, and a clear improvement in exercise tolerance was observed in some subjects, as measured by $6 \mathrm{MWD}$ or Peak $\dot{\mathrm{V}} \mathrm{O}_{2}$. As the magnitude of clinically relevant change in these measures has, in some cases, been reported to be $\pm 5 \%$ for LVEF, \pm 1 class for NYHA classification, $\pm 50 \mathrm{~m}$ for $6 \mathrm{MWD}$, and $\pm 1.5 \mathrm{ml} \cdot \mathrm{kg}^{-1} \cdot \mathrm{min}^{-1}$ for Peak $\dot{\mathrm{V}}{ }_{2},{ }^{13}$ not a few subjects experienced a clinically relevant improvement in this study.

Validity of Efficacy Endpoints LVEF is the most common measure used in clinical practice and has been commonly used as an outcome measure in clinical studies. However, patients with severe HF are more likely to have comorbid mitral insufficiency or renal impairment and changes in preload and afterload are caused by various triggers despite administration of diuretics. Assessment of HF status by LVEF alone, a measure that is affected by preload and afterload, is therefore difficult. In addition, the possibility of measurement bias by an assessor cannot be ruled out.

In consideration of the current situation that patients with symptoms of HF in the absence of decreased LVEF, such as patients with $\mathrm{HF}$ with preserved $\mathrm{EF}$, account for half of all patients with $\mathrm{HF},{ }^{14}$ assessment of cardiac function in patients with HF should include changes in diastolic function. Data from a clinical study conducted by Osaka University, Japan, showed that a review of symptoms and exercise tolerance in patients without LV reverse remodeling found an improvement in pulmonary arterial hypertension and a decrease in pulmonary vascular resistance, as determined by cardiac catheterization. However, data on measures to assess cardiac function needs to be accumulated. Conventional endpoints for regenerative medicine products include improvement in LV contractility as measured by various modalities, which now serves as an efficacy endpoint. ${ }^{3}$ In recent years, the US Food and Drug Administration has stated that LV contractility cannot be used as an efficacy endpoint in confirmatory studies, which should use endpoints such as mortality and cardiovascular or HF hospitalizations. ${ }^{15}$ In addition, the Study Group of the Japan's Ministry of Health, Labour and Welfare reported that improvement in survival outcomes and quality of life should be used as efficacy outcome measures. ${ }^{16}$ With regard to study design, whether a double- or single-arm design should be used for evaluation is controversial. However, based on the abovementioned suggestions and the results of this clinical study, the efficacy of regenerative medicine products likely cannot be adequately evaluated using LV contractility alone as an efficacy outcome measure. Therefore, efficacy assessment from diversified perspectives is considered necessary. ${ }^{17}$

As described earlier, improvement in survival outcomes or prevention of $\mathrm{HF}$ hospitalizations is important in terms of maintenance or improvement of HF status. The previous clinical study conducted by Osaka University reported a survival rate $91.7 \%$ at 3 years after transplantation in 17 patients who received transplantation with the skeletal myoblast sheet. The event-free rate at 3 years after transplantation based on Kaplan-Meier curves using the endpoints of death, LVAS implantation for worsening cardiac function, and dependence on catecholamine agents was $85.7 \%$, suggesting a higher event-free rate than that in patients who are registered for heart transplantation at Osaka University and waiting for transplantation as Status 2 patients (54.3\%), and these data suggest the efficacy of the skeletal myoblast sheet. Although the evaluation period of 26 weeks in this study still makes any discussion difficult, we await the results from an ongoing follow-up study of up to 2 years after transplantation.

\section{Safety}

In this study, no deaths occurred during the 26-week evaluation period and none of the subjects withdrew from the study because of ADRs after transplantation of TCD-51073. In contrast, SAEs included prolonged HF in all the subjects at 31 days after transplantation and, additionally, aggravated HF in 1 subject at 49 days after transplantation. The events of prolonged and aggravated HFs were attributed to the transplantation procedure and underlying disease, respectively. Both were reported during hospitalization after transplantation, suggesting that surgical indication in patients with severe HF should be carefully considered, including postoperative management.

\section{Arrhythmia}

Since cases of serious arrhythmia were reported in a clinical study of suspension of skeletal myoblasts injected into the myocardium, ${ }^{18}$ the potential arrhythmogenic effects of skeletal myoblast transplantation have been discussed. In contrast, animal experiments have demonstrated that skeletal myoblast sheets are free of such side effects. ${ }^{19}$ In the present study, no events of serious arrhythmia were observed and no significant change in the incidence of ventricular extrasystole was found on Holter ECG monitoring. Therefore, the arrhythmogenic risk from using TCD-51073 was considered low.

\section{Study Design}

As transplantation of TCD-51073 involves invasive surgery, inclusion of a concurrent control group in clinical studies that include subjects with severe disease, such as the present study, is difficult for ethical reasons. Patients with severe symptoms in the present study had already received maximum therapies and had no further treatments for HF. Such patients expect the possible efficacy of this treatment and so all want to receive this treatment and none want to enter the control group. Although a placebo effect is expected to occur when evaluating efficacy before and after therapy in study patients in single-arm efficacy studies, the future development of new efficacy outcome measures that are unaffected by the placebo effect may promote the establishment of an efficacy assessment system that is ethically appropriate and provides highly reliable data.

\section{Significance of TCD-51073 in the Treatment of HF}

Although findings from previous clinical research studies and clinical trials suggest that TCD-51073 is unlikely to achieve a marked improvement in cardiac contractility in patients with severe HF who are receiving maximal standard-of-care therapy, this product may be a potential alternative therapy for $\mathrm{HF}$, other than the currently available standardized therapies, such as drug therapy, LVAS implantation, and heart transplantation, if improvement of clinical symptoms, improved exercise tolerance, prolonged survival, and consequent improved cardiac diastolic function are demonstrated in future studies. A current concern in the treatment of HF is the absolute shortage of donors, and we believe that using this product as a bridge to LVAS or heart transplantation to suppress the progress of HF and delay the timing of LVAS implantation while applying various measures to increase donors will contribute to the resolution of the donor shortage. In addition, a reduction in the number of HF hospitalizations and the length of hospital stay because of HF with the use of this product will be beneficial in reducing the escalation of medical costs. 


\section{Study Limitations}

This study has the following limitations: (1) a small sample size, which is inadequate for statistical analysis; (2) transplantation of TCD-51073 occurred in the absence of other concomitant therapies, so an add-on effect is unlikely to occur in the before-and-after comparisons; however, comparison with a group of untreated patients is necessary; (3) single-arm, open-label study, so the possibility of assessment bias cannot be ruled out; an independent analysis should be considered; and (4) the evaluation period of 26 weeks after transplantation is limited for demonstrating the efficacy of TCD-51073 in improving survival outcomes and preventing hospitalizations because of $\mathrm{HF}$, thus requiring further mid- and long-term follow-up data.

\section{Conclusions}

This study demonstrated the feasibility and safety of the transplantation of TCD-51073 in patients with severe chronic HF due to ischemic heart disease, suggesting that TCD-51073 might maintain or improve cardiac function, symptoms, and physical function.

\section{Acknowledgments}

We thank all of the investigators and site staff who participated in this clinical study.

\section{Disclosures}

UMIN study Identifier: UMIN000008013. Sponsor/Name of Grant: Terumo Corporation.

All authors serve as investigators in this trial. Y. Sawa has received joint research funds provided by Terumo Corporation. K.K. is an endowed chair sponsored by Terumo Corporation.

\section{References}

1. Imamura $\mathrm{T}$, Kinugawa $\mathrm{K}$, Hatano $\mathrm{M}$, Fujino $\mathrm{T}$, Inaba $\mathrm{T}$, Maki H, et al. Status 2 patients had poor prognosis without mechanical circulatory support. Circ J 2014; 78: 1396-1404.

2. Sakaguchi T, Matsumiya G, Yoshioka D, Miyagawa S, Nishi H, Yoshikawa $\mathrm{Y}$, et al. DuraHeart ${ }^{\mathrm{TM}}$ magnetically levitated left ventricular assist device: Osaka University experience. Circ J 2013; 77: $1736-1741$.

3. Sanganalmath SK, Bolli R. Cell therapy for heart failure: A comprehensive overview of experimental and clinical studies, current challenges, and future directions. Circ Res 2013; 113: 810-834.

4. Dib N, McCarthy P, Campbell A, Yeager M, Pagani FD, Wright S, et al. Feasibility and safety of autologous myoblast transplantation in patients with ischemic cardiomyopathy. Cell Transplant 2005; 14: $11-19$.

5. Duckers HJ, Houtgraaf J, Hehrlein C, Schofer J, Waltenberger J, Gershlick A, et al. Final results of a phase IIa, randomised, openlabel trial to evaluate the percutaneous intramyocardial transplantation of autologous skeletal myoblasts in congestive heart failure patients: The SEISMIC trial. EuroIntervention 2011; 6: 805-812.

6. Menasché P, Alfieri O, Janssens S, McKenna W, Reichenspurner H, Trinquart L, et al. The Myoblast Autologous Grafting in Ischemic Cardiomyopathy (MAGIC) trial: First randomized placebo-controlled study of myoblast transplantation. Circulation 2008; 117: $1189-1200$.

7. Miyagawa S, Saito A, Sakaguchi T, Yoshikawa Y, Yamauchi T, Imanishi $\mathrm{Y}$, et al. Impaired myocardium regeneration with skeletal cell sheets: A preclinical trial for tissue-engineered regeneration therapy. Transplantation 2010; 90: 364-372.

8. Kondoh H, Sawa Y, Miyagawa S, Sakakida-Kitagawa S, Memon IA, Kawaguchi N, et al. Longer preservation of cardiac performance by sheet-shaped myoblast implantation in dilated cardiomyopathic hamsters. Cardiovasc Res 2006; 69: 466-475.

9. Hata H, Matsumiya G, Miyagawa S, Kondoh H, Kawaguchi N, Matsuura N, et al. Grafted skeletal myoblast sheets attenuate myocardial remodeling in pacing-induced canine heart failure model. $J$ Thorac Cardiovasc Surg 2006; 132: 918-924.

10. Memon IA, Sawa Y, Fukushima N, Matsumiya G, Miyagawa S, Taketani S, et al. Repair of impaired myocardium by means of implantation of engineered autologous myoblast sheets. $J$ Thorac Cardiovasc Surg 2005; 130: 1333-1341.

11. Shudo Y, Miyagawa S, Nakatani S, Fukushima S, Sakaguchi T, Saito A, et al. Myocardial layer-specific effect of myoblast cell-sheet implantation evaluated by tissue strain imaging. Circ J 2013; 77: $1063-1072$.

12. Sawa Y, Miyagawa S, Sakaguchi T, Fujita T, Matsuyama A, Saito A, et al. Tissue engineered myoblast sheets improved cardiac function sufficiently to discontinue LVAS in a patient with DCM: Report of a case. Surg Today 2012; 42: 181-184.

13. Jessup M, Greenberg B, Mancini D, Cappola T, Pauly DF, Jaski B, et al; Calcium Upregulation by Percutaneous Administration of Gene Therapy in Cardiac Disease (CUPID) Investigators. Calcium Upregulation by Percutaneous Administration of Gene Therapy in Cardiac Disease (CUPID): A phase 2 trial of intracoronary gene therapy of sarcoplasmic reticulum $\mathrm{Ca}^{2+}$-ATPase in patients with advanced heart failure. Circulation 2011; 124: 304-313.

14. Shiba N, Nochioka K, Miura M, Kohno H, Shimokawa H; CHART-2 Investigators. Trend of westernization of etiology and clinical characteristics of heart failure patients in Japan: First report from the CHART-2 study. Circ J 2011; 75: 823-833.

15. US Food and Drug Administration. Guidance for industry: Cellular therapy for cardiac disease. FDA, MD, October 2010. Available from www.fda.gov [Guidance, Compliance \& Regulatory Information] (accessed April 9, 2015).

16. Revision of "Guidelines on Clinical Evaluation of Drugs to Treat Heart Failure", Notification No. 0329 [18] of the Evaluation and Licensing Division, Pharmaceutical and Food Safety Bureau, March 29, 2011. Available from: http://www.pmda.go.jp/files/000157362. pdf (accessed April 9, 2015).

17. Hare JM, Bolli R, Cooke JP, Gordon DJ, Henry TD, Perin EC, et al. Cardiovascular Cell Therapy Research Network. Phase II clinical research design in cardiology: Learning the right lessons too well: Observations and recommendations from the Cardiovascular Cell Therapy Research Network (CCTRN). Circulation 2013; 127: $1630-1635$.

18. Menasché $\mathrm{P}$, Hagège AA, Vilquin JT, Desnos M, Abergel E, Pouzet B, et al. Autologous skeletal myoblast transplantation for severe postinfarction left ventricular dysfunction. J Am Coll Cardiol 2003; 41: $1078-1083$.

19. Narita T, Shintani Y, Ikebe C, Kaneko M, Harada N, Tshuma N, et al. The use of cell-sheet technique eliminates arrhythmogenicity of skeletal myoblast-based therapy to the heart with enhanced therapeutic effects. Int J Cardiol 2013; 168: 261-269.

\section{Supplementary Files}

Supplementary File 1

Table S1. Inclusion and exclusion criteria for study of cell sheet transplantation

Table S2. Observation and test items and schedule of study of cell sheet transplantation

Figure S1. Serial change in B-type natriuretic peptide from baseline to 26 weeks after cell sheet transplantation.

Please find supplementary file(s);

http://dx.doi.org/10.1253/circj.CJ-15-0243 